\title{
RGAugury: a pipeline for genome-wide prediction of resistance gene analogs (RGAs) in plants
}

\author{
Pingchuan Li ${ }^{1}$, Xiande Quan ${ }^{1}$, Gaofeng Jia ${ }^{1,2}$, Jin Xiao ${ }^{1,3}$, Sylvie Cloutier ${ }^{4}$ and Frank M. You ${ }^{1 *}$
}

\begin{abstract}
Background: Resistance gene analogs (RGAs), such as NBS-encoding proteins, receptor-like protein kinases (RLKs) and receptor-like proteins (RLPS), are potential R-genes that contain specific conserved domains and motifs. Thus, RGAs can be predicted based on their conserved structural features using bioinformatics tools. Computer programs have been developed for the identification of individual domains and motifs from the protein sequences of RGAs but none offer a systematic assessment of the different types of RGAs. A user-friendly and efficient pipeline is needed for large-scale genome-wide RGA predictions of the growing number of sequenced plant genomes.

Results: An integrative pipeline, named RGAugury, was developed to automate RGA prediction. The pipeline first identifies RGA-related protein domains and motifs, namely nucleotide binding site (NB-ARC), leucine rich repeat (LRR), transmembrane (TM), serine/threonine and tyrosine kinase (STTK), lysin motif (LysM), coiled-coil (CC) and Toll/ Interleukin-1 receptor (TIR). RGA candidates are identified and classified into four major families based on the presence of combinations of these RGA domains and motifs: NBS-encoding, TM-CC, and membrane associated RLP and RLK. All time-consuming analyses of the pipeline are paralleled to improve performance. The pipeline was evaluated using the well-annotated Arabidopsis genome. A total of 98.5, 85.2, and $100 \%$ of the reported NBS-encoding genes, membrane associated RLPs and RLKs were validated, respectively. The pipeline was also successfully applied to predict RGAs for 50 sequenced plant genomes. A user-friendly web interface was implemented to ease command line operations, facilitate visualization and simplify result management for multiple datasets.
\end{abstract}

Conclusions: RGAugury is an efficiently integrative bioinformatics tool for large scale genome-wide identification of RGAs. It is freely available at Bitbucket: https://bitbucket.org/yaan/pc/rgaugury.

Keywords: Resistance gene analog (RGA), Nucleotide binding site (NBS), Receptor like protein (RLP), Receptor like kinase (RLK), Genome-wide prediction, Pipeline

\section{Background}

Aside from physical and chemical barriers, plants protect themselves from pathogen infections by employing a sophisticated biochemical immune system composed mainly of two layers. The first layer is conferred by cell surface pattern-recognition receptors (PRRs) that detect general elicitors pathogen/microbe-associated molecule patterns (PAMP/MAMPs), known as PAMP-triggered immunity (PTI). The defence could be overcome by specific pathogen effectors $[1,2]$. Plants have also evolved

\footnotetext{
* Correspondence: Frank.You@agr.gc.ca

'Morden Research and Development Centre, Agriculture and Agri-Food

Canada, Morden, MB R6M 1Y5, Canada

Full list of author information is available at the end of the article
}

other types of receptors called resistance $(R)$ proteins that recognize specific effectors and elicit a robust counter-attack system termed effector-triggered immunity (ETI) [3]. This second response layer corresponds to a gene-for-gene interaction [4].

Resistance gene analogs (RGAs) comprise both PRRs and $R$-genes and, most have conserved domains and motifs [5]. The majority of characterized PRRs are either surface-localized receptor-like protein kinases (RLKs) or membrane associated receptor-like proteins (RLPs) [6-8]. RLKs possess an extracellular sensing domain, a transmembrane (TM) region and an intracellular protein kinase, containing two types according to the domain structure, leucine rich repeat (LRR) type, such as FLS2 [9], 
EFR [10] and XA21 [11], and, lysin motif (LysM)-type, such as CERK1 [12]. RLPs have similar domain architecture to RLKs except for the absence of a kinase domain in their intracellular region [13], such as Cf-9 (LRR-type) [14], Eix1 and Eix2 (LRR-type) [15], CEBiP (LysM-type) [16]. R-proteins or effector-recognition receptors are described as intracellular immune receptors and most belong to nucleotide-binding site-LRR (NBS-LRR or NLR) class [17]. Seven domains or motifs may be found in R-proteins: Toll/Interleukin-1 receptor (TIR), coiled-coil (CC), leucine zipper (LZ), NBS, LRR, TM and serinethreonine kinase (STK) [18]. Based on these domains, R-proteins are categorized into five main classes: (1) CC-NBS-LRR (CNL), (2) TIR-NBS-LRR (TNL), (3) RLKs, (4) RLPs and (5) other variants [19].

Pyramiding of plant resistance genes in new cultivars is the most effective and environmentally friendly approach for plant disease control and reduction of yield losses. Development of diagnostic molecular markers associated with disease resistance is a prerequisite for molecular resistance breeding. Marker saturation in the vicinity of the target resistance gene is a critical step for mapped-based or positional cloning of R-genes which results in the development of diagnostic markers [20]. RGAs-based marker development strategies have been successfully applied for the development of diagnostic markers for orange wheat blossom midge and wheat stem rust resistance genes [21, 22]. This strategy involves four iterative steps: (1) identification of genome-wide RGAs, (2) identification of potential RGA candidates in the vicinity of the target resistance gene using comparative genomics analysis, (3) design of SNP markers for candidate RGAs, and (4) marker evaluation using biparental genetic populations and/or association panels. Therefore, the identification of genome-wide RGAs is a useful genomic resource for fine-mapping and cloning of resistance genes and, for marker development for resistance breeding.

To date, hundreds of NBS-LRR, RLK and RLP genes have been reported in plants [13, 23-27]. In these reports, RGAs were detected using several individual computing programs to predict related domains and/or motifs followed by manual or semi-automated summarization of the results with custom scripts. These programs include BLAST [28], Hmmer3 [29], InterProScan5 with Pfam and SMART databases [30, 31], Phobius [32], TMHMM [33], pfam_scan [34], and nCoils [35]. The diversity of tools and parameters used in these studies makes it impossible to properly compare their outputs [5]. In addition, tools, such as nCoils, Phobius and pfam_scan, do not have multiple-threading features or are not optimized for large datasets, limiting their performance in genome-wide analyses. As the number of sequenced genomes increase, rapid and accurate RGA identification will benefit genome annotation endeavours. Here, we developed a comprehensive pipeline to address these shortfalls. Our primary objective was to develop an efficient and integrative pipeline tool to identify all known types of RGA candidates from genome-scale datasets, with a user-friendly interface and, that would be fully integrated from dataset to summarization. The pipeline, which we named RGAugury, was validated using the well-annotated Arabidopsis genome data and successfully applied to 50 sequenced genomes for comparative analysis.

\section{Implementation}

Pipeline design

NBS and TM-CC containing proteins and membrane associated RLPs and RLKs belong to the main four known RGA families [5], and were thus included in the current version of RGAugury. RGA identification generally includes two basic steps: the identification of all conserved domains and/or motifs from protein sequences translated from gene sequences, followed by the analysis of the domain and/or motif composition including the classification of the genes into one of the four known types of RGAs based on such domain and/or motif structures. To improve the computing performance for the genome-wide identification of RGAs, three additional solutions were implemented in the pipeline: (1) an initial filtering of RGA candidates to remove a large portion of nonRGA genes, (2) parallel computing for time-consuming calculations, and (3) selection of the most efficient protein databases for domain detection. The design and the workflow of the pipeline are summarized in Fig. 1 and described in the following sections.

\section{Initial filtering of RGA candidates}

Because RGAs occupies a small percentage of the total genes in a genome, initial filtering to remove non-RGA genes can dramatically reduce the number of genes for the downstream domain/motif detection which saves considerable computing time. To do so, we used BLASTP to identify potential RGA candidates against an RGA database called RGAdb (see description below). The BLAST+ package was selected for sequence alignment as it outperforms BLAST in calculation power under the same conditions [36]. In the initial step, the input protein sequences are aligned against RGAdb by BLASTP using an E-value cut-off of 1e-5 (this E-value cut-off may be adjusted according to different species). Non-RGA proteins are filtered out (Fig. 1). Based on the analyses of 50 sequenced plant genomes, an average of $76.4 \%$ of the annotated genes were removed, significantly abridging the downstream analysis time.

RGAdb was constructed using protein sequences and their annotations from the NCBI non-redundant protein database (nr) (http://www.ncbi.nlm.nih.gov/), plant 


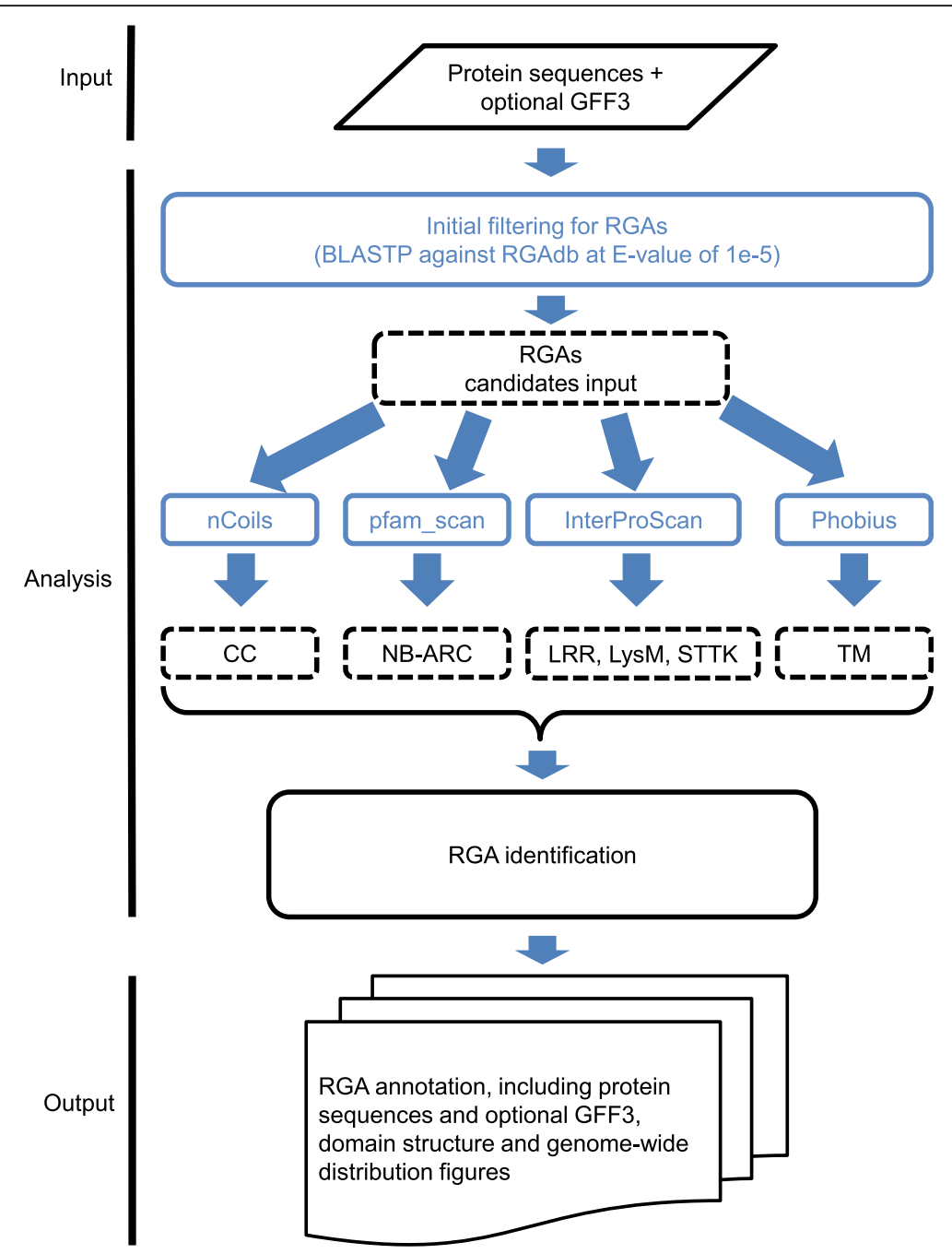

Fig. 1 Workflow of RGAugury. The pipeline was designed to use protein sequences to detect conserved domains and motifs found in genes involved in plant resistance and identify RGAs by integrating results generated from five programs: BLAST, InterProScan, pfam_scan, nCoil and Phobius. The annotated candidates for four different RGA types are exported as plain files. Analyses performed in parallel mode are labelled in blue. Intermediate results are indicated by a dashed-line box. GFF3: Generic Feature Format version 3; CC: coiled-coil; LRR: leucine-rich repeat; NB-ARC: nucleotide binding adapter shared by APAF-1, R gene products and CED-4; STTK: serine/threonine and tyrosine kinase; LysM: lysin motif; TM: transmembrane

resistance gene database (PRGdb, http://www.prgdb.org) [19]) and other cloned $R$-genes. Sequence entries were retrieved from $n r$ with the key words 'resistance' and 'disease'. Disease-irrelevant entries such as resistance to aluminum, drought, cold, DNA-damage, herbicide and UVB were removed. A total of 14,906 disease resistance related sequences were retained. In addition, 'contributed,' 'putative' and 'references' entries from PRGdb were merged and the resulting dataset was filtered to remove redundant and non-annotated entries. As a result, 44,109 entries from PRGdb were appended into RGAdb. A few NBS encoding proteins from wheat and Brachypodium were also added into RGAdb [37-39]. Finally RGAdb contains 59,597 entries which were derived from more than 300 plant species (Additional file 1) and $80 \%$ of the database entries are NBS coding proteins, RLKs, RLPs, MLO- and RPW-like proteins. In addition, this database will be regularly maintained to remove irrelevant entries and add new RGA entries.

\section{Domain and motif detection}

The initial filtering results in a reduced set of potential RGA candidates for the downstream domain and motif detection. There are seven RGAs-related domains and motifs, including NB-ARC or NBS, LRR, TM, STTK, LysM, CC and TIR in proteins. To detect them, four third-party tools were chosen and integrated into the pipeline. nCoils program was used to identify the $\mathrm{CC}$ 
domain present in CNL, $\mathrm{CN}$ and TM-CC types [35]. Minor modifications on the original source code of nCoils were made under its redistribution permission to facilitate the RGAugury pipeline to call nCoils through command-line. For detection of the NBARC domain, the pfam_scan toolkit was preferred because it outperformed InterProScan according to the test results obtained using the Arabidopsis genome data (data not shown). In addition, unlike InterProScan, the $P$-value parameter in pfam_scan is adjustable offering flexibility for the adjustment of the $P$-value cut-off across plant species. However, InterProScan was chosen to identify LRR and LysM, which are two components of RLPs and RLKs that play a role in pathogen signal recognition [16]. InterProScan is also suitable for the detection of the STTK domains of RLKs [25, 40-42]. Two tools, Phobius [32] and TMHMM [33], are available for TM domain identification but Phobius was elected because it performed better than TMHMM [32]. RGAugury's configuration file can be easily modified to add any additional domains or motifs for detection, thus offering flexibility and extendibility.

InterProScan is a protein domain identifier that uses up to 14 databases for detection [43]. The accuracy and computing performance depend on the number and nature of the databases used for analysis. Pfam, Gene3D, SMART, Superfamily and the external database Panther were chosen for RGA domain detection based on accuracy and computing performance for the detection of LRR, LysM and STTK domains/motifs (data not shown). Three modes for database selection are provided in the pipeline. The default 'Quick' mode uses only Pfam and Gene3D which can identify most domains and motifs from input protein sequences. In the 'Deep' mode, Superfamily and SMART are also included for domain detection. As a consequence of the additional databases, the 'Deep' mode may perform slightly more slowly but this reduction in performance must be weighed against the accuracy and completeness of the results coveted. Differences between the two modes were mainly in the numbers of identified proteins with LRR motifs. The third mode is called 'Free' mode. This mode allows users to select one or more databases from a list. This mode is usually intended for result confirmation but can also be of use in custom applications. Here, the Panther (Protein Analysis Though Evolutionary Relationships) [44] database is introduced to the pipeline. Panther is a large protein database for comprehensive protein evolutionary and functional classification that contains more than 12,000 protein families from 104 sequenced genomes [45]. Our test results indicated that Panther would require huge calculation resource upon CPU threads for genome-scale protein analysis, which may take days to weeks depending on the input sequences. Thus, it is not suitable for genomescale RGA identification but it can be accessed in the 'Free' mode for specific purposes such as the confirmation of small numbers of identified RGA candidates for example.

\section{RGA identification}

Once all domains and motifs are identified from the input protein sequences, RGAugury creates a table that lists the RGA candidates initially identified by BLASTP, along with their identified domains and coordinates on genes. The RGA identification module was developed to classify genes as potential RGAs and to classify them into specific RGA families (Fig. 2). First, the program classifies genes containing an NB-ARC or a TIR domain into the NBS encoding family. Genes that contain a TM domain may belong to the RLP, RLK or TM-CC families depending on the presence of other domains in the gene structures. If no NB-ARC, TIR or TM is observed, the gene is discarded as a non-RGA. The NBS-encoding gene family members are further divided into several subgroups according to their domain architecture, namely NBS, CNL, TNL, TN, CN, NL, TX and OTHER that may have chimeric domain/motif architecture. Here $\mathrm{C}, \mathrm{N}, \mathrm{L}, \mathrm{T}$ and $\mathrm{X}$ represent $\mathrm{CC}$, NBS or NB-ARC, LRR, TIR and unknown domains, respectively. For example, a gene with CC, NB-ARC and LRR domains is classified in the CNL subgroup. However, a gene with both TIR and CC domains ends up in the OTHER subgroup. In this case, a user needs to manually check this unexpected domain combination using the exported domain information. A gene with only an NB-ARC domain would require confirmation by InterProScan.

\section{Data input and result output}

The command-line pipeline requires three parameters. The first parameter is a protein sequence file in FASTA format from either a whole genome annotation project or manually annotated protein sequence data. The second one is the $P$-value cut-off for the initial RGA filtering using BLASTP. The default value is set to $1 \mathrm{e}-5$. The last parameter informs on the database(s) to be queried for domain detection using InterProScan. RGAugury provides an optional parameter for specifying a companion Generic Feature Format (GFF3) or Gene Transfer Format (GTF) file when they are available. GFF3 or GTF files are helpful to draw RGA distribution plots using CViT [46] and draw gene and RGA domain structure plots. For most sequenced plant genomes, GFF3 annotation files can be downloaded from Phytozome [47]. 


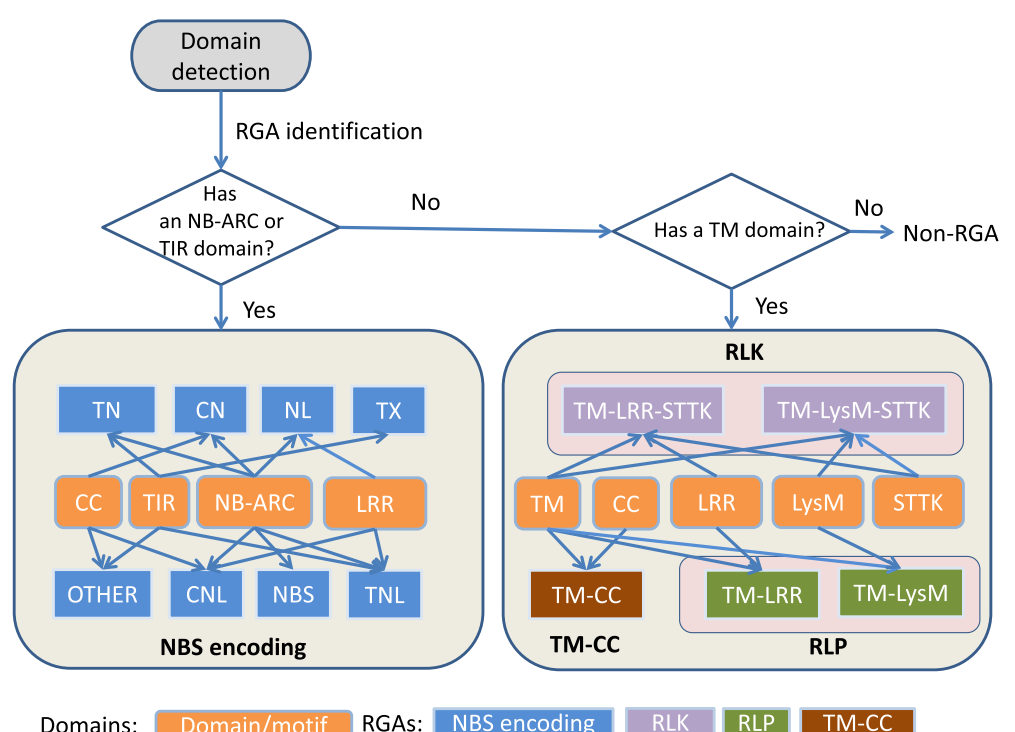

Fig. 2 RGA identification based on domain structures of genes. CC: coiled-coil; CN: CC-NBS; CNL: CC-NBS-LRR; LRR: leucine rich repeat; LysM: lysin motif; NB-ARC: nucleotide binding site-activity regulated cytoskeleton; NBS: nucleotide-binding site; NL: NBS-LRR; RGA: resistance gene analog; RLK: receptor like kinase; RLP: receptor like protein; STTK: serine/threonine and tyrosine kinase; TIR: Toll/Interleukin-1 receptor; TM: transmembrane; TN: TIR-NBS; TNL: TIR-NBS-LRR; TX: TIR-unknown domain

Processing files derived from domain detection step and final RGA identification results are exported to plain text files. When a GFF file is provided, distribution plots of RGA families on chromosomes and the gene and domain structures for each RGA candidate are also generated.

\section{Implementation of the command-line version}

A single command-line pipeline program was written in Perl to seamlessly process all analyses. As domain detection for genome-scale protein sequences is computationally intensive, performance was a critical factor considered during the design of RGAugury. Benefitting from the development of programming techniques and hardware, parallel computing can significantly boost large-scale data analysis. To parallel heavy analyses, a Perl module, tool.pm, which invokes the fork function to parallel the execution of BLAST searches in the initial RGA filtering and domain detection, was implemented.

\section{Web interface}

To ease the command-line operations, a user-friendly web interface was designed and implemented using PHP and Java script (Fig. 3a). The necessary FASTA formatted protein sequences can be copied and pasted into the sequence text box or uploaded from a sequence file. A GFF3 file corresponding to the input protein sequences is optional but recommended. Databases for InterProScan can be selected through one of three modes: 'Quick,' 'Deep' or 'Free' (flexible selection of up to five available databases).
The default $P$-value cut-off for initial RGA filtering was set to 1 e- 5 but can be altered at user's wish.

A project name is used as an intuitive ID for project management purposes. A 'Job List Status' page is dynamically generated upon project submission or following a mouse click on the 'Status of Submitted Projects' link (Additional file 2: Figure S1A). Finished jobs and current status of jobs being processed are displayed. Finished job are marked with the status "complete" in green that can be updated with the "Check" link to the "Results and Summaries' page that shows the summary information, such as the numbers of RGAs and the detailed domain structure thumbnails for individual RGAs (Fig. 3b). The 'Results and Summaries' page also provides a link to download intermediate and final result files. All RGA candidates listed can be browsed. A search box is provided to query the candidates using full or partial letters or numbers from the table. For each RGA, an individual page will be generated by clicking on the Gene Structure Viewer (GSV) icon to show the details of the gene structure (Additional file 2: Figure S1B). GSV also provides links to the EnsemblePlants and GenBank websites for the genes that are available in these two databases, a useful feature for accessing additional gene information. When a GFF3 file is submitted along with the protein sequences, figures of RGA distribution on chromosomes are shown by clicking on the 'Whole genome RGA distribution can be viewed' link on Results and Summaries page (Additional file 2: Figure S1C).

The web version of RGAugury provides a new feature for InterProScan version check. When InterProScan 


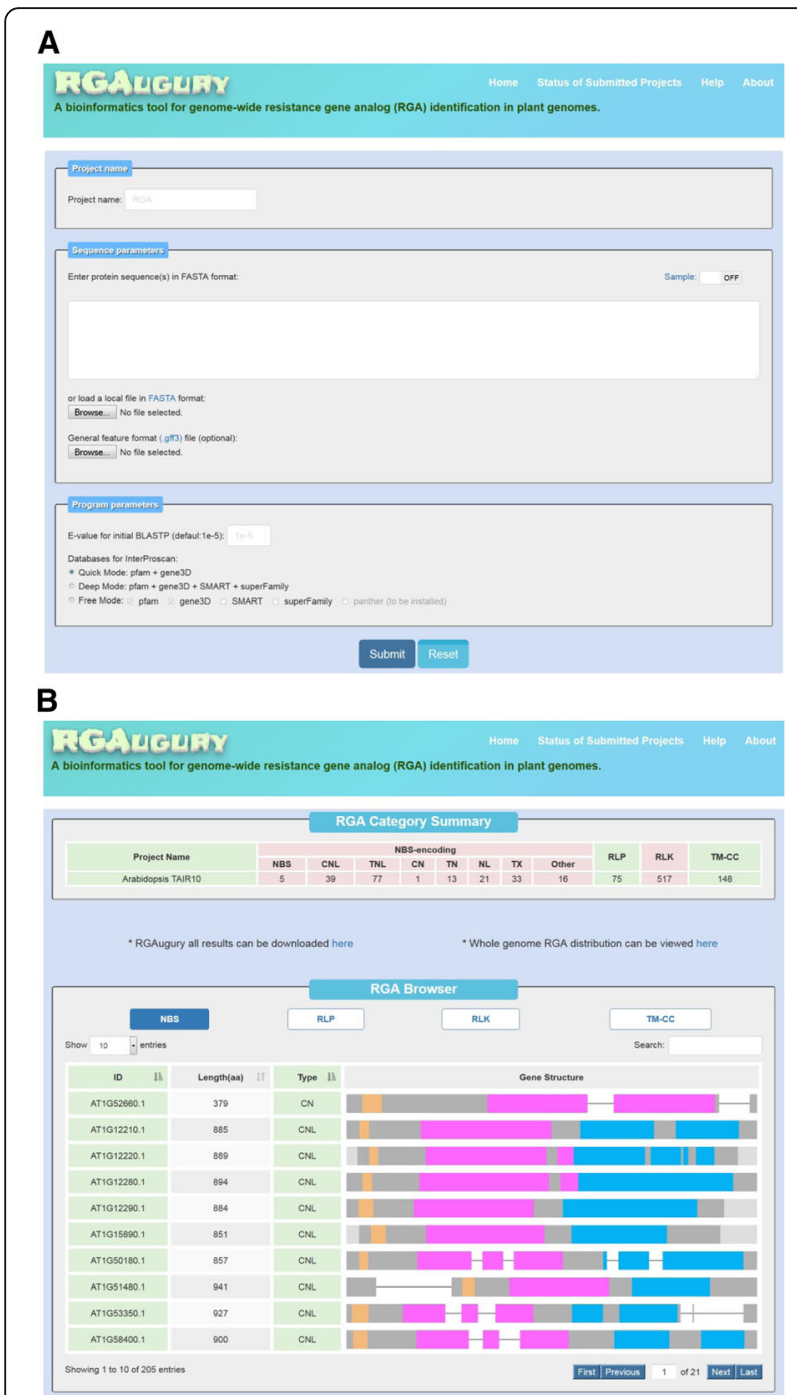

Fig. 3 Web user interface pages of RGAugury. a The main page of RGAuguary for data input. All parameter values required in the command line version are specified directly on this page. Only protein sequences in FASTA format are required. A GFF3 file corresponding to the input protein sequences is optional but recommended. Databases for InterProscan can be selected by choosing either a predesigned 'Quick' mode or a 'Deep' mode. The default E-value cut-off for the initial RGA filtering with BLASTP is 1e-5. b The RGA prediction result summary page

runs on a web server, the web program will automatically check over the Internet whether the local InterProScan and its domain databases are up-to-date. If not, the web program pop-up dialog box reminding the user to update the software and its databases. In addition, detailed help pages are provided and synced with the RGAugury Bitbucket Wiki page (https://bitbucket.org/ yaanlpc/rgaugury/wiki). Any updates on the Wiki page will also be reflected on the web help pages.

The RGAugury web program provides a project management function. Users can cancel executing jobs or delete finished projects from the server. Overall, the web version of RGAugury provides flexibility, convenience and interactive functionality over the command-line pipeline without compromising the RGA identification capabilities. The RGAugury web program can run on an HTTP server such as Apache. As hours of computing time are needed to finish a genome-scale RGA prediction, RGAugury is not suited for installation on a public web server. However, users are advised to download the pipeline and its web program from Bitbucket (https:// bitbucket.org/yaanlpc/rgaugury) and install them in users' local servers which can be used through intranet.

\section{Result and discussion RGA identification accuracy}

Case studies were performed to test the prediction accuracy of the RGAugury pipeline. The protein sequences of the well annotated Arabidopsis genome (TAIR 10) [13, 25] were used for analysis. A total of 207 NBS-encoding genes were previously reported [27] but 14 of them were later considered rejected in TAIR10 as a consequence of erroneous annotations. The remaining 193 genes were used as a test data set for NBS-encoding genes. All protein sequences were downloaded from the NIBLRRS project website (http://niblrrs.ucdavis.edu). A total of 190 (98.5\%) out of 193 NBS-containing proteins were predicted and validated by the RGAugury pipeline (Table 1, Additional file 2). The three proteins with inconsistent prediction were AT5G45510, AT5G17950 and AT3G26470. AT5G45510 and AT5G17950 had an LRR domain but lacked an NB-ARC domain, while AT3G26470 was reported as $R P W 8$, an oth-R type of resistance gene [48] that was presumed to have a TM and a CC domains rather than a NB-ARC domain [49]. As such, these three genes may have been originally incorrectly annotated [27]. If this inference is true, then the prediction accuracy of RGAugury is $100 \%$ for NBS encoding genes in Arabidopsis. To further test RGAugury's ability to detect genes harboring NBS motifs, all 27,416 unique Arabidopsis protein sequences (TAIR10) were processed through the pipeline. Besides the same 190 proteins mentioned above, an additional 15 NBSencoding genes were predicted (Additional file 2). Of these newly predicted RGA genes, 6 NB-ARC domain

Table 1 Evaluation of RGA identification accuracy with RGAugury using the Arabidopsis thaliana dataset (TAIR10)

\begin{tabular}{lllc}
\hline RGA type & No. of known RGAs & No. of RGAs identified & \% identified \\
\hline NBS & 193 & 190 & 98.5 \\
RLP & 54 & 46 & 85.2 \\
RLK & 456 & 460 & 100.0 \\
\hline
\end{tabular}


containing genes were already annotated as disease resistance genes in TAIR10, the remaining 9 TX type genes were classified as unknown functions.

A similar evaluation was performed for RLP- and RLK-type RGAs. The previous report [50] indicated that the Arabidopsis genome encodes 57 RLPs, of which three have no sequence in TAIR 10, precluding their further analysis. Out of the remaining 54 RLPs, 46 (85.2 \%) were predicted and 8 remained unclassified as a consequence of the absence of a TM domain (Table 1, Additional file 3). For RLKs, a total of 608 members were reported in the Arabidopsis genome, but only $75 \%$ (456) of them were claimed to be associated with membranes [25]. RGAugury validated all 456 membrane-associated RLKs and identified additional four RLKs (Table 1, Additional file 4). The recently cloned wheat stem rust disease resistance genes $s r 33$ and $s r 35$ were also validated by RGAugury as NBS-LRR resistance genes [37, 38]. These case studies demonstrate that RGAugury accurately predicts RGAs on a genome-wide scale, and can be used for $R$-gene annotation.

\section{Computing performance and multiple thread optimization}

RGAugury can run in either 'Quick' or 'Deep' mode which rely on the number of databases used for domain detection. Differences between the two modes resided mostly in the numbers of identified LRR containing proteins (Table 2). For example, LOC_Os11g42590.1, an NL type in rice, was identified as NBS type using the 'Quick' mode but as an NL type using the 'Deep' mode. The LRR domain in this gene can be identified by either Superfamily or SMART database through InterProScan. However, these differences were minor in most cases (Table 2). Thus, the 'Quick' mode is recommended for fast RGA annotation.

Though BLASTP has its own multiple thread parameter $(-n)$, the paralleled tool, tool.pm, developed herein, outperformed it. Two threading methods were compared using 40 CPUs. BLASTP searches were performed for 5000 and 10,000 protein sequences randomly selected from the Arabidopsis genome against RGAdb using two threading methods (Additional file 5). An improvement of 3.8- and 3.4-fold in execution speed for the two datasets, respectively, was observed for the tool.pm module when compared to the thread parameter $(-n)$ of BLASTP. Speed improvement of the domain detection programs nCoil, Phobius and pfam_scan theoretically depends on the called CPU numbers because they lack embedded multiple threads function. Thus, application of tool.pm to these programs significantly reduces the time needed for domain detection, making it possible to shorten computing time for genome-scale RGA identification to mere hours.
To further evaluate the performance of RGAugury, we predicted RGAs for 50 sequenced plant genomes, including 44 angiosperm species and 6 green algae. All protein sequences were downloaded from the Phytozome (ver11.0) database website [47]. With both 'Quick' and 'Deep' modes, the processing time was highly correlated with the number of coding genes in genomes $\left(R^{2}=0.76\right.$ for the 'Quick' mode and 0.75 for the 'Deep' mode) (Fig. 4). The total processing time averaged $2.4 \mathrm{~h}$ in 'Quick' mode. The 'Deep' mode averaged an extra $0.5 \mathrm{~h}$ but resulted in the enhanced prediction of LRR domains in some cases. Maize (Zea mays) data was not included in the correlation analysis because only $15.9 \%$ of the input protein sequences were retained after the initial BLATP filtering compared with an average of $23.6 \%$ among these 50 plant genomes, resulting in significantly fewer hours needed for the downstream domain identification. In general, RGAugury saves an average of $76 \%$ time in the downstream domain/motif analysis if the initial BLASTP filtering step is performed before domain detection, demonstrating that the RGAugury pipeline is efficient for large-scale genome-wide RGA identification.

\section{Large-scale genome-wide RGA identification for compara- tive analysis}

A summary of the RGA prediction results for the 50 sequenced plant genomes is shown in Table 2. NBS encoding genes are divided into two major groups based on their variable N-terminals: TIR and non-TIR [51]. It has been hypothesized that TNL have never evolved in monocots [52] or have been discarded during evolution [51, 53-55]. Tarr et al. [56] developed an indirect method to search for TNL in monocots and magnoliids based on degenerate PCR and, they did not observed any TNL in the studied monocots. Among the 50 genomes studied here, nine belong to three different monocot orders: Poales (Brachypodium distachyon, Oryza sativa, Panicum hallii, Panicum virgatum, Setaria italic, Sorghum bicolor, Z. mays), Zingiberales (Musa acuminata) and Alismatales (Spirodela polyrhiza). Our RGAugury prediction of these monocot species did not identify any TNL type confirming the previous findings (Table 2). Genes encoding TN or TX proteins were detected in these monocots but the numbers were smaller compared to genes predicted to encode other types of NBS motifs and compared to those from species other than monocots, implying that TNL may have been discarded during the evolution in the monocot lineage.

No genes coding for NB-ARC domains were predicted from the model green algae Chlamydomonas reinhardtii, Volvox carteri, and Cyanidioschyzon merolae because TIR and non-TIR NBS encoding genes arose only in the Plantae [57]. Our prediction results for all six green algae organisms confirmed this observation (Table 2), 
Table 2 Summary of RGA identification results for 50 sequenced plant genomes

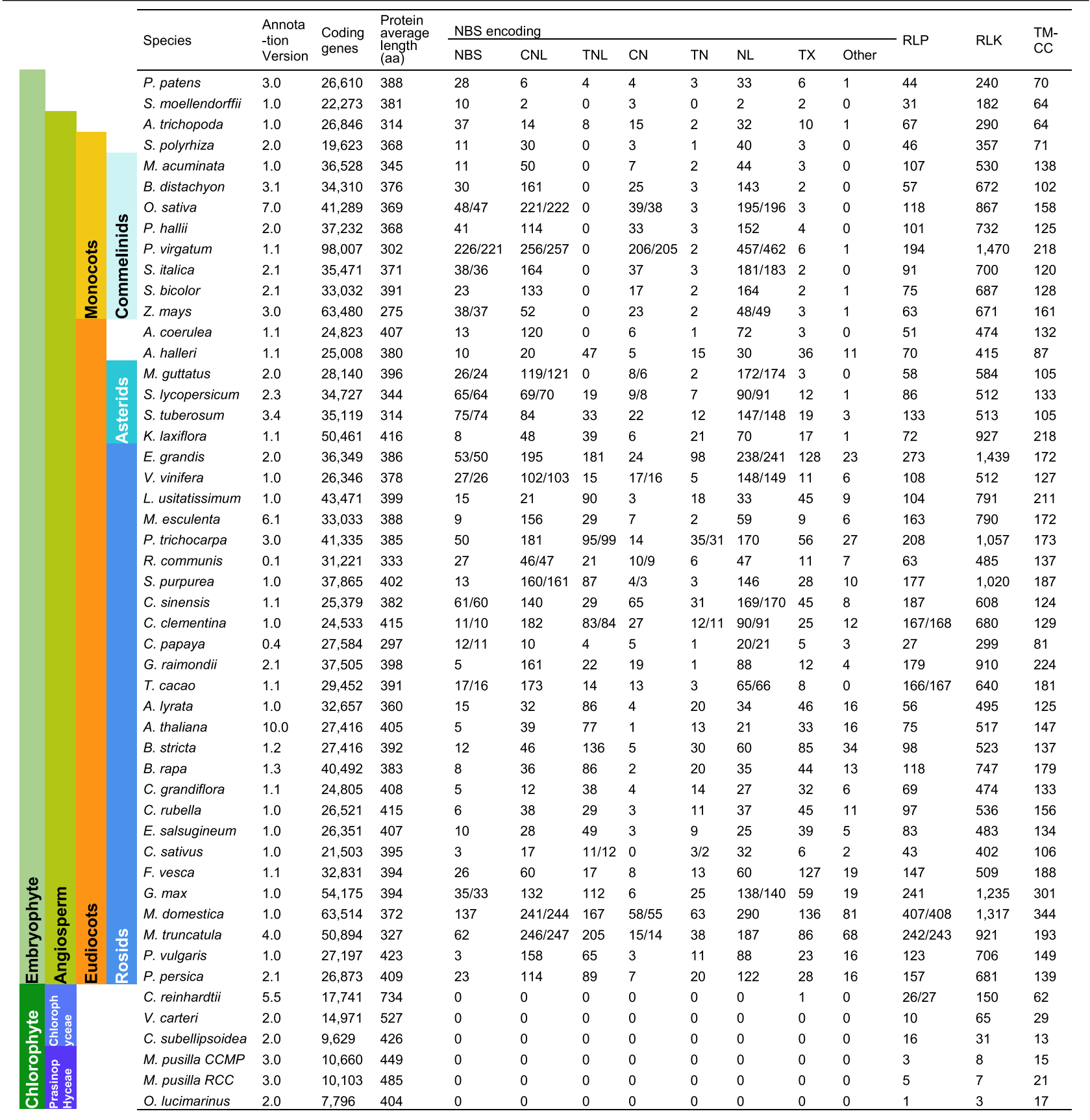

Note: Two modes for database selection were used: the 'Quick' mode (Pfam + Gene3D) and the 'Deep' mode (Pfam + Gene3D + SMART + Superfamily). Results were separated by a slash if differences existed between the two modes. Plants were sorted by taxonomic groups which are labelled on the left side of the table. $A$. coerulea: Aquilegia coerulea; A. halleri: Anemone halleri; A. lyrata: Arabidopsis lyrata; A. thaliana: Arabidopsis thaliana; A. trichopoda: Amborella trichopoda; $B$. distachyon: Brachypodium distachyon; B. rapa: Brassica rapa; B. stricta: Boechera stricta; C. clementina: Citrus clementina; C. grandiflora: Capsella grandiflora; C. papaya: Carica papaya; C. reinhardtii: Chlamydomonas reinhardtii; C. rubella: Capsella rubella; C. sativus: Cucumis sativus; C. sinensis: Citrus sinensis; C. subellipsoidea: Coccomyxa subellipsoidea; E. grandis: Eucalyptus grandis; E. salsugineum: Eutrema salsugineum; F. vesca: Fragaria vesca; G. max: Glycine max; G. raimondii: Gossypium raimondii; K. laxiflora: Kalanchoe laxiflora ; L. usitatissimum: Linum usitatissimum; M. acuminata: Musa acuminata; M. domestica: Malus domestica; M. esculenta: Manihot esculenta; M. guttatus: Mimulus guttatus; M. pusilla: Micromonas pusilla; M. truncatula: Medicago truncatula; $O$. lucimarinus: Ostreococcus lucimarinus; $O$. sativa: Oryza sativa; P. hallii: Panicum hallii; P. patens: Physcomitrella patens; P. persica: Prunus persica; P. trichocarpa: Populus trichocarpa; P. virgatum: Panicum virgatum; P. vulgaris: Phaseolus vulgaris; R. communis: Ricinus communis; S. bicolor: Sorghum bicolor; S. italica: Setaria italica; S. lycopersicum: Solanum lycopersicum; S. moellendorffii: Selaginella moellendorffii; S. polyrhiza: Spirodela polyrhiza; S. purpurea: Salix purpurea; S. tuberosum: Solanum tuberosum; T. cacao: Theobroma cacao; V. carteri: Volvox carteri; V. vinifera: Vitis vinifera; Z. mays: Zea mays 


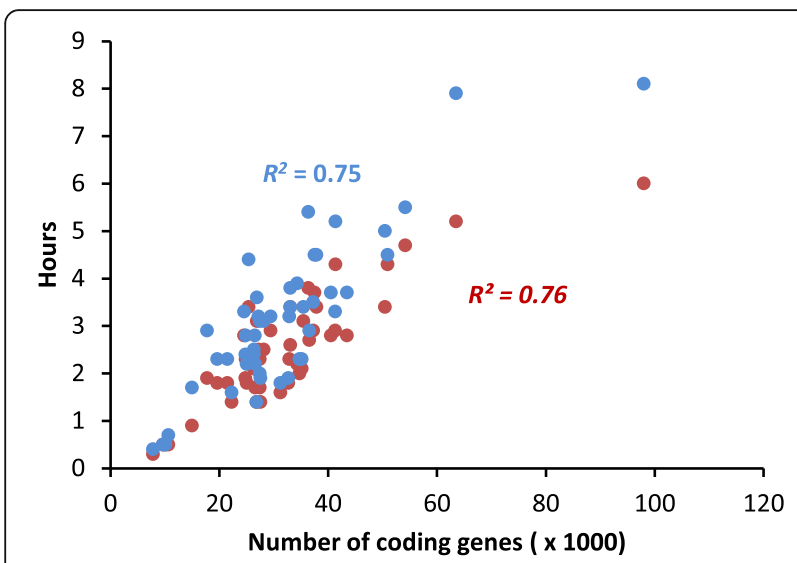

Fig. 4 Performance of RGAugury. Forty-nine sequenced plant genomes (Zea mays was excluded, see text) with varying numbers of protein coding genes were used for RGA identification on a server embedded with 40 CPUs. Time to complete the processing of the entire pipeline for each dataset was recorded as a performance measurement. Performance for the 'Quick' mode (Pfam + Gene3D databases) and 'Deep' mode (Pfam + Gene3D + SMART + Superfamily) were compared. The dots and $R^{2}$ value in red represent results for the 'Quick' mode and those in red represent the results for the 'Deep' mode

providing additional validation for the RGAugury prediction ability.

\section{Conclusions}

We developed a command-line and a web version of an integrative, efficient and user-friendly pipeline called RGAugury, for large-scale genome-wide RGA prediction based on translated protein sequences. Four types of RGAs, namely NBS-encoding, RLP, RLK and TM-CC can be predicted. The results from validation data sets and the 50 sequenced plant genomes demonstrated its high accuracy and utility.

\section{Additional files}

Additional file 1: Contents of the RGAdb database. (XLSX $31 \mathrm{~kb}$ )

Additional file 2: Figure S1. Additional web user interface pages of RGAugury. (A) The Status of Submitted Projects page. (B) Gene and domain structure page for an identified RGA. Detailed information is included in a spreadsheet result file. External links to NCBI and EnsemblPlants are indicated with their respective website logo. (C) RGA distribution on chromosomes. NBS encoding, RLP, RLK and TM-CC RGAs are represented by different color bars. ALL represents a combined RGA distribution figure for the merged data of all four RGA families. (PPTX $359 \mathrm{~kb}$ )

Additional file 3: Prediction results for NBS-encoding genes in the Arabidopsis genome. (XLSX $58 \mathrm{~kb}$ )

Additional file 4: Prediction results for RLP genes in the Arabidopsis genome. (XLSX $11 \mathrm{~kb}$ )

Additional file 5: Prediction results for RLK genes in the Arabidopsis genome. (XLSX $21 \mathrm{~kb}$ )

\section{Abbreviations}

CC: Coiled-coil; CN: CC-NBS; CNL: CC-NBS-LRR; ETI: Effector-triggered immunity; GFF: Generic feature format; GPL: GNU general public license;
GSV: Gene structure viewer; GTF: Gene transfer format; LRR: Leucine rich repeat; LysM: Lysin motif; LZ: Leucine zipper; NB-ARC: Nucleotide binding adaptor shared by APAF-1, certain $R$ gene products and CED-4; NBS: Nucleotide-binding site; NL: NBS-LRR; PAMP/MAMP: Pathogen/microbeassociated molecule pattern; PRR: Pattern-recognition receptor; PTI: PAMP triggered immunity; RGA: Resistance gene analog; RLK: Receptor like kinase; RLP: Receptor like protein; STK: Serine-threonine kinase; STTK: Serine/ threonine and tyrosine kinase; TIR: Toll/Interleukin-1 receptor;

TM: Transmembrane; TN: TIR-NBS; TNL: TIR-NBS-LRR; TX: TIR-unknown domain

\section{Acknowledgement}

None.

\section{Funding}

The research was supported by the GRDI program (project No. J-000006) funded by Agriculture and Agri-food Canada.

\section{Availability of data and materials}

RGAugury is released under open source GPL license. The command line pipeline and its web version can be downloaded from Bitbucket: https:// bitbucket.org/yaanlpc/rgaugury. The detailed instructions for installation and usages are described in its help pages. A simple demo for RGAugury is also provided at http://rgaugury.bitbucket.org/ for viewing only.

\section{Authors' contributions}

FY and SC planned the work. PL carried out the research, command line pipeline implementation, web user interface design, and drafted the manuscript. XQ and GJ implemented the web version of the pipeline. JX provided insights and revised the manuscript. FY supervised the study and revised the manuscript. SC revised the manuscript. All authors read and approved the final manuscript.

\section{Competing interests}

The authors declare that they have no competing interests.

\section{Consent for publication}

Not applicable.

Ethics approval and consent to participate

Not applicable.

\section{Author details}

${ }^{1}$ Morden Research and Development Centre, Agriculture and Agri-Food Canada, Morden, MB R6M 1Y5, Canada. ${ }^{2}$ University of Saskatchewan, Saskatoon, SK S7N 5A8, Canada. ${ }^{3}$ National Key Laboratory of Crop Genetics and Germplasm Enhancement, Cytogenetics Institute, Nanjing Agricultural University, Nanjing 210095, China. ${ }^{4}$ Ottawa Research and Development Centre, Agriculture and Agri-Food Canada, Ottawa, ON K1A 0C6, Canada.

Received: 9 August 2016 Accepted: 25 October 2016

Published online: 02 November 2016

\section{References}

1. Gohre V, Spallek T, Haweker H, Mersmann S, Mentzel T, Boller T, et al. Plant pattern-recognition receptor FLS2 is directed for degradation by the bacterial ubiquitin ligase AvrPtoB. Curr Biol. 2008;18(23):1824-32.

2. Macho AP, Zipfel C. Targeting of plant pattern recognition receptortriggered immunity by bacterial type-III secretion system effectors. Curr Opin Microbiol. 2015;23:14-22.

3. Jones JD, Dangl JL. The plant immune system. Nature. 2006;444(7117):323-9.

4. Van der Biezen EA, Jones JD. Plant disease-resistance proteins and the gene-for-gene concept. Trends Biochem Sci. 1998;23(12):454-6.

5. Sekhwal MK, Li P, Lam I, Wang X, Cloutier S, You FM. Disease resistance gene analogs (RGAs) in plants. Int J Mol Sci. 2015;16(8):19248-90.

6. Böhm H, Albert I, Fan L, Reinhard A, Nürnberger T. Immune receptor complexes at the plant cell surface. Curr Opin Plant Biol. 2014;20:47-54

7. Zipfel C. Plant pattern-recognition receptors. Trends Immunol. 2014;35(7):345-51.

8. Monaghan J, Zipfel C. Plant pattern recognition receptor complexes at the plasma membrane. Curr Opin Plant Biol. 2012;15(4):349-57. 
9. Gomez-Gomez L, Boller T. FLS2: an LRR receptor-like kinase involved in the perception of the bacterial elicitor flagellin in Arabidopsis. Mol Cell. 2000;5(6):1003-11.

10. Zipfel C, Kunze G, Chinchilla D, Caniard A, Jones JD, Boller T, et al. Perception of the bacterial PAMP EF-Tu by the receptor EFR restricts Agrobacterium-mediated transformation. Cell. 2006;125(4):749-60.

11. Song WY, Wang GL, Chen LL, Kim HS, Pi LY, Holsten T, et al. A receptor kinase-like protein encoded by the rice disease resistance gene, $\mathrm{Xa21}$. Science. 1995;270(5243):1804-6.

12. Shimizu T, Nakano T, Takamizawa D, Desaki $Y$, Ishii-Minami N, Nishizawa $Y$, et al. Two LysM receptor molecules, CEBiP and OsCERK1, cooperatively regulate chitin elicitor signaling in rice. Plant J. 2010;64(2):204-14.

13. Fritz-Laylin LK, Krishnamurthy N, Tor M, Sjolander KV, Jones JD. Phylogenomic analysis of the receptor-like proteins of rice and Arabidopsis. Plant Physiol. 2005:138(2):611-23.

14. Jones DA, Thomas CM, Hammond-Kosack KE, Balint-Kurti PJ, Jones JD. Isolation of the tomato Cf-9 gene for resistance to Cladosporium fulvum by transposon tagging. Science. 1994;266(5186):789-93.

15. Ron M, Avni A. The receptor for the fungal elicitor ethylene-inducing xylanase is a member of a resistance-like gene family in tomato. Plant Cell. 2004;16(6):1604-15.

16. Kaku H, Nishizawa Y, Ishii-Minami N, Akimoto-Tomiyama C, Dohmae N, Takio K, et al. Plant cells recognize chitin fragments for defense signaling through a plasma membrane receptor. Proc Natl Acad Sci U S A. 2006;103(29):11086-91.

17. Kim J, Lim CJ, Lee BW, Choi JP, Oh SK, Ahmad R, et al. A genome-wide comparison of NB-LRR type of resistance gene analogs (RGA) in the plant kingdom. Mol Cells. 2012;33(4):385-92.

18. van Ooijen G, van den Burg HA, Cornelissen BJ, Takken FL. Structure and function of resistance proteins in solanaceous plants. Annu Rev Phytopathol. 2007:45:43-72.

19. Sanseverino W, Roma G, De Simone M, Faino L, Melito S, Stupka E, et al. PRGdb: a bioinformatics platform for plant resistance gene analysis. Nucleic Acids Res. 2010;38(Database issue):D814-821.

20. Jander G, Norris SR, Rounsley SD, Bush DF, Levin IM, Last RL. Arabidopsis map-based cloning in the post-genome era. Plant Physiol. 2002;129(2):440-50.

21. Kassa MT, Haas S, Schliephake E, Lewis C, You FM, Pozniak CJ, et al. A saturated SNP linkage map for the orange wheat blossom midge resistance gene Sm1. Theor Appl Genet. 2016;129(8):1507-17.

22. Kassa MT, You FM, Fetch TG, Fobert P, Sharpe A, Pozniak CJ, et al. Genetic mapping of SrCad and SNP marker development for marker-assisted selection of Ug99 stem rust resistance in wheat. Theor Appl Genet. 2016;129(7):1373-82

23. Jia J, Zhao S, Kong X, Li Y, Zhao G, He W, et al. Aegilops tauschii draft genome sequence reveals a gene repertoire for wheat adaptation. Nature. 2013:496(7443):91-5

24. Lozano R, Hamblin MT, Prochnik S, Jannink JL. Identification and distribution of the NBS-LRR gene family in the Cassava genome. BMC Genomics. 2015;16:360.

25. Shiu SH, Karlowski WM, Pan R, Tzeng YH, Mayer KF, Li WH. Comparative analysis of the receptor-like kinase family in Arabidopsis and rice. Plant Cell. 2004;16(5):1220-34.

26. Chen JY, Huang JQ, Li NY, Ma XF, Wang JL, Liu C, et al. Genome-wide analysis of the gene families of resistance gene analogues in cotton and their response to Verticillium wilt. BMC Plant Biol. 2015;15:148.

27. Meyers BC, Kozik A, Griego A, Kuang H, Michelmore RW. Genome-wide analysis of NBS-LRR-encoding genes in Arabidopsis. Plant Cell. 2003;15(4):809-34.

28. Camacho C, Coulouris G, Avagyan V, Ma N, Papadopoulos J, Bealer K, et al. BLAST+: architecture and applications. BMC Bioinformatics. 2009;10:421.

29. Eddy SR. Accelerated profile HMM searches. PLoS Comput Biol. 2011;7(10): e1002195.

30. Schultz J, Copley RR, Doerks T, Ponting CP, Bork P. SMART: a web-based tool for the study of genetically mobile domains. Nucleic Acids Res. 2000;28(1):231-4

31. Zdobnov EM, Apweiler R. InterProScan-an integration platform for the signature-recognition methods in InterPro. Bioinformatics. 2001;17(9):847-8.

32. Kall L, Krogh A, Sonnhammer EL. A combined transmembrane topology and signal peptide prediction method. J Mol Biol. 2004;338(5):1027-36.

33. Krogh A, Larsson B, von Heijne G, Sonnhammer EL. Predicting transmembrane protein topology with a hidden Markov model: application to complete genomes. J Mol Biol. 2001;305(3):567-80.
34. Finn RD, Mistry J, Tate J, Coggill P, Heger A, Pollington JE, et al. The Pfam protein families database. Nucleic Acids Res. 2010;38(Database issue):D211-222.

35. Lupas A, Van Dyke M, Stock J. Predicting coiled coils from protein sequences. Science. 1991;252(5009):1162-4.

36. Zhang Z, Schwartz S, Wagner L, Miller W. A greedy algorithm for aligning DNA sequences. J Comput Biol. 2000;7(1-2):203-14.

37. Periyannan S, Moore J, Ayliffe M, Bansal U, Wang X, Huang L, et al. The gene Sr33, an ortholog of barley Mla genes, encodes resistance to wheat stem rust race Ug99. Science. 2013;341(6147):786-8.

38. Saintenac C, Zhang W, Salcedo A, Rouse MN, Trick HN, Akhunov E, et al. Identification of wheat gene Sr35 that confers resistance to Ug99 stem rust race group. Science. 2013;341(6147):783-6.

39. Tan S, Wu S. Genome wide analysis of nucleotide-binding site disease resistance genes in Brachypodium distachyon. Comp Funct Genomics. 2012:2012:418208.

40. Shiu SH, Bleecker AB. Plant receptor-like kinase gene family: diversity, function, and signaling. Sci STKE. 2001;2001(113):re22.

41. Shiu SH, Bleecker AB. Receptor-like kinases from Arabidopsis form a monophyletic gene family related to animal receptor kinases. Proc Natl Acad Sci U S A. 2001;98(19):10763-8.

42. Shiu SH, Bleecker AB. Expansion of the receptor-like kinase/Pelle gene family and receptor-like proteins in Arabidopsis. Plant Physiol. 2003;132(2):530-43.

43. Quevillon E, Silventoinen V, Pillai S, Harte N, Mulder N, Apweiler R, et al. InterProScan: protein domains identifier. Nucleic Acids Res. 2005;33(Web Server issue):W116-120.

44. Thomas PD, Kejariwal A, Campbell MJ, Mi H, Diemer K, Guo N, et al. PANTHER: a browsable database of gene products organized by biological function, using curated protein family and subfamily classification. Nucleic Acids Res. 2003;31(1):334-41.

45. Mi H, Poudel S, Muruganujan A, Casagrande JT, Thomas PD. PANTHER version 10: expanded protein families and functions, and analysis tools. Nucleic Acids Res. 2016:44(D1):D336-342.

46. Cannon EK, Cannon SB. Chromosome visualization tool: a whole genome viewer. Int J Plant Genomics. 2011:2011:373875.

47. Goodstein DM, Shu S, Howson R, Neupane R, Hayes RD, Fazo J, et al. Phytozome: a comparative platform for green plant genomics. Nucleic Acids Res. 2012;40(Database issue):D1178-1186.

48. Sanseverino W, Ercolano MR. In silico approach to predict candidate R proteins and to define their domain architecture. BMC Res Notes. 2012;5:678.

49. Xiao S, Ellwood S, Calis O, Patrick E, Li T, Coleman M, et al. Broad-spectrum mildew resistance in Arabidopsis thaliana mediated by RPW8. Science. 2001:291(5501):118-20

50. Wang G, Ellendorff U, Kemp B, Mansfield JW, Forsyth A, Mitchell K, et al. A genome-wide functional investigation into the roles of receptor-like proteins in Arabidopsis. Plant Physiol. 2008;147(2):503-17.

51. Meyers BC, Dickerman AW, Michelmore RW, Sivaramakrishnan S, Sobral BW, Young ND. Plant disease resistance genes encode members of an ancient and diverse protein family within the nucleotide-binding superfamily. Plant J. 1999;20(3):317-32

52. Akita M, Valkonen JP. A novel gene family in moss (Physcomitrella patens) shows sequence homology and a phylogenetic relationship with the TIRNBS class of plant disease resistance genes. J Mol Evol. 2002;55(5):595-605.

53. Cannon SB, Zhu H, Baumgarten AM, Spangler R, May G, Cook DR, et al. Diversity, distribution, and ancient taxonomic relationships within the TIR and non-TIR NBS-LRR resistance gene subfamilies. J Mol Evol. 2002;54(4):548-62.

54. Bai J, Pennill LA, Ning J, Lee SW, Ramalingam J, Webb CA, et al. Diversity in nucleotide binding site-leucine-rich repeat genes in cereals. Genome Res. 2002:12(12):1871-84.

55. Pan Q, Wendel J, Fluhr R. Divergent evolution of plant NBS-LRR resistance gene homologues in dicot and cereal genomes. J Mol Evol. 2000;50(3):203-13.

56. Tarr DE, Alexander HM. TIR-NBS-LRR genes are rare in monocots: evidence from diverse monocot orders. BMC Res Notes. 2009;2:197.

57. Yue JX, Meyers BC, Chen JQ, Tian D, Yang S. Tracing the origin and evolutionary history of plant nucleotide-binding site-leucine-rich repeat (NBS-LRR) genes. New Phytol. 2012;193(4):1049-63. 